\title{
Clinical study of student learning using mastery style versus immediate feedback online activities
}

\author{
Gary Gladding, Brianne Gutmann, Noah Schroeder, ${ }^{*}$ and Timothy Stelzer \\ Department of Physics, University of Illinois Urbana Champaign, Urbana, Illinois 61801, USA
}

(Received 9 December 2014; published 1 June 2015)

\begin{abstract}
This paper is part of a series of studies to improve the efficacy of online physics homework activities by integrating narrated animated solutions with mastery inspired exercises. In a clinical study using first- and second-year university students, the mastery group attempted question sets in four levels, with animated solutions between each attempt, until mastery was achieved on each level. This combined elements of formative assessment, the worked example effect, and mastery learning. The homework group attempted questions with immediate feedback and unlimited tries. The two groups took a similar amount of time to complete the activity. The mastery group significantly outperformed the homework group on a free response post-test that required students to show their work in solving near and far transfer problems.
\end{abstract}

DOI: 10.1103/PhysRevSTPER.11.010114

PACS numbers: 01.40.- $\mathrm{d}, 01.50 .-\mathrm{i}$

\section{INTRODUCTION}

Online platforms for delivering homework have become ubiquitous in science classes. For example, WebAssign states that over one million students across 2,300 institutions use their platform every year [1]. Computer based homework systems have been studied for more than 40 years with mixed performance results in direct comparisons between paper and online homework systems [2-4]. Online systems allow efficient distribution of homework to many students, and also allow instructors to decide whether to give feedback immediately, that is, upon each answer submission, or to delay the feedback. Evidence exists supporting improved learning from immediate feedback [5], as well as delayed feedback [6]. A 2008 review by Shute found that the timing of feedback should be tailored to the skills being taught, recommending immediate feedback for procedural tasks, and delayed feedback for simpler tasks to promote transfer of learning [7]. Butler and Roediger, in a 2008 paper using cued recall of passages, found that giving correctness feedback after a test was completed resulted in greater recall than giving feedback after each item in a test [8]. There does not appear to be a one-size-fits-all result for feedback timing.

Another benefit of online homework systems is that instructors can easily make material available to students, including both practice problems and worked examples. Teaching strategies that stress the use of worked examples have been shown to be more effective than problem solving in areas like middle school mathematics [9], algebra [10],

\section{schroe16@illinois.edu}

Published by the American Physical Society under the terms of the Creative Commons Attribution 3.0 License. Further distribution of this work must maintain attribution to the author(s) and the published article's title, journal citation, and DOI. and physics [11]. Novice students prefer this method of instruction as well $[12,13]$. Cognitive load theory states that, when solving problems, novices may use up cognitive resources employing costly means-end analysis techniques, hampering the acquisition of schemas [14]. These kinds of behaviors include attempting many combinations of equations with relevant variables in order to find the correct answer to a problem. Immediate feedback style homework allows for this strategy by not penalizing students for wrong answers. Students can make many attempts without assessing whether the strategy used to get the answer is sound. Worked examples show novice students expertlike strategies, and the increased learning from this technique is known as the worked example effect [15]. However, detailed instructions can hamper more experienced expert students' ability to learn from examples [16], distracting these students from forming patterns, and not taking advantage of their ability to form sets of steps into chunks [17]. Online homework systems can also deliver animated worked examples to students, as opposed to static solutions on paper. Worked examples can be designed to reduce cognitive load [18], and as animation technology has progressed, so has research into multimedia content delivery $[19,20]$. For example, multimedia learning modules have been shown to be more effective than textbooks in introducing new materials $[21,22]$.

Online homework systems also provide flexibility in content delivery. Instructors can opt for formats other than simply rewarding course credit to students for completing a set number of questions correctly. One such option is mastery learning, whereby students are required to attain a certain level of success in a topic or skill before moving on in the assignment. Bloom's Learning for Mastery (1968) outlines a model for mastery learning that stresses formative evaluation of student skills to help diagnose students' weaknesses, and alternative learning resources provided by 
teachers to help students achieve mastery if they have not yet done so [23]. Past analyses of mastery learning have found positive effects when comparing mastery learning to conventional homework [24], with some evidence that weaker students may benefit more from mastery style instruction [25].

We will compare the traditional online homework format, which utilizes immediate correctness feedback and unlimited tries to correctly answer questions, to a mastery setting, where students are given a set of questions to answer, and are only given correctness feedback after the entire set is completed. Narrated animated solutions videos are also provided between attempts for the mastery group, and students are challenged to answer new versions of the question sets until they demonstrate mastery by answering all questions correctly. Our goals were to study differences in student interaction with the two different homework formats, as well as to assess how much students learned from the two homework styles via a post-test.

We chose to investigate this model of instruction because it integrates many effects that have independently been shown to help students learn, including the worked example effect [15], multimedia learning [21], the testing effect [26], transfer appropriate processing [27], and formative assessment [28]. The narrated animated solutions we use take advantage of the worked example and multimedia learning effects. This benefit is usually not present in traditional online homework. Additionally, the increased number of questions attempted by students during the mastery exercises increases benefits from the testing effect. The students are allowed one chance to answer questions in the mastery assessments, which is more examlike than multiattempt online homework. This provides students with frequent realistic formative assessment and prepares them for future exams via transfer appropriate processing. Mastery learning incorporates these effects and will help students apply concepts and skills consistently, and retrieve them successfully later on in the course.

This experiment is a follow-up to an experiment that investigated student learning from mastery style cycles of questions and solutions [29]. This experiment uses the same materials as the previous experiment, but the organization of the materials has been changed. The previous experiment found that students learned from the solution videos and outperformed a control group that did not receive any instruction on a post-test.

\section{POPULATION}

Participants in the experiment were enrolled in the calculus-based University Physics: Electricity \& Magnetism course at the University of Illinois at Urbana-Champaign. This is the second physics course required for physics and engineering majors and is usually taken during the second year at university. No credit was offered to the students for this experiment, but they were told that if they completed the experiment, they would have access to an extra office hour provided by experienced TAs. A total of 126 students signed up. These students were randomly split into two treatment groups, a mastery group and a homework group. Of the students that signed up for the experiment, 32 from the Mastery group and 27 from the homework group participated in the experiment.

\section{PROCEDURE AND MATERIALS}

The entire experiment was completed by each student in one sitting. Students in our experiment were separated into two treatment groups, the "Mastery" group and the "Homework" group. Both groups progressed through four topic levels: (i) Electric fields from infinite sheets of charge, (ii) surface charge densities in planar geometries, (iii) electric fields and single-region potential differences with spherical charges, and (iv) multiregion potential differences with spherical charges. The mastery group experienced the learning materials in a mastery setting, characterized by four elements: (1) Students were required to answer all questions in a level before getting any feedback. (2) Feedback included correctness as well as a narrated animated solution video outlining a correct solution strategy for each individual question. (3) Students were required to answer all questions in a level correctly before moving to the next level. (4) If a student failed to answer all questions correctly, after availing themselves of the feedback, students would attempt a new version of the level, with questions similar but not identical to the initial version, repeating the process until the level is mastered. For example, mastery students began with access to level 1 only, which consisted of four questions on superposition of electric fields with planar charge sheets and conducting slabs. Three of these questions required an algebraic solution, and the final was a numeric calculation. Students submitted answers for all four questions, and then had all four questions simultaneously graded. Correctness feedback was given, and students were given the opportunity to view solutions for all questions, those answered both correctly and incorrectly. The solutions were created and animated using Powerpoint graphics and animation, and were narrated and recorded using TechSmith's Jing recording software [30]. Solutions were designed with multimedia principles in mind, heavily influenced by the work of Mayer [19] and Chen [31]. Screenshots of the final frame of some representative solutions are included in the Appendix [32]. After indicating that they were finished viewing the solutions, access to the solutions was removed and the student was given their next set of questions. Students that failed to answer all questions correctly attempted a new version of level 1 , with new charge layouts as well as a shift in the aspect of the system to be calculated, such as changing the region in which they are asked to calculate the electric field. Students that succeeded in answering all questions correctly, mastering the material, moved on to the second level, consisting of four questions 
related to surface charge densities. Three of the questions asked for a condition that would give rise to a particular field, and the other asked for a numeric calculation of a surface charge density. This was followed by a third level of five numeric calculation questions that covered calculating the potential difference when given the functional form of the electric field, basic electric fields in spherical geometries, as well as single-integral potential differences in that geometry. The final level of four questions covered multiple-integral potential differences, including two numeric calculations of a potential difference when given a piecewise defined electric field, one numeric calculation of a potential difference that crossed a charged shell, and a conceptual question considering how potential difference changed if the geometry of a situation was changed. For the first, third, and fourth levels four versions of questions were used. The second topic used three versions of questions. Because of the high penalty of having to do 4 new questions if one was answered incorrectly, we chose to give the questions in a multiple choice format. We hoped this would reduce false negatives, as the system could not account for students who knew how to do the question well but did not get the correct final number due to a careless mistake. The penalty for doing so for the mastery group, new versions of each question, was much harsher when compared to the homework group, which could re-examine work to find small errors and quickly resubmit an answer. The question sets are attached in the Appendix. Students worked on the mastery materials, mastering them in order, until they finished the fourth mastery set. If a student requested to end the instructional portion of the experiment and move to the post-test, they were allowed to do so.

The second group, the Homework group, was given the same initial version of questions for each of the 4 levels seen by the mastery group, but the format was modified to simulate immediate feedback homework given to students in online homework systems in four ways: (i) All numeric questions were given as free response questions instead of multiple choice. (ii) Students were given immediate correctness feedback for their answer to each question. (iii) Students could retry a question as many times as necessary to get it correct, and incorrect responses did not result in a new version of a question, and (iv) students had access to all questions, and could answer them in any order they chose. The third condition necessitated that solution videos were not available to students in the homework group, as that would have given away the answer. To further mimic the online homework environment, students were encouraged to work together with other participants, and use resources such as prelecture videos and lecture notes, as well as any online resources they could find. Ten of the 17 questions asked for numeric answers, and to simulate online homework, they were given in a free response format. The other 7 had an answer that was either symbolic or a conditional statement, and a multiple-choice format was used. Students worked on the homework style questions until they completed them or until they elected to take the post-test.

A post-test was given to assess how well students in both groups learned the skills required to complete the levels in the learning materials, and how well they could use that knowledge in new situations. The post-test consisted of ten questions that all pertained to potential difference, ranging from questions very similar to the learning materials (calculating the potential difference with spherical charges) to questions that were much more different (applying potential difference skills learned in spherical geometries to electric fields learned in planar geometries). The post-test is included in the Appendix. The post-test was a standard paper based exam and students were asked to show their work. All post-tests were graded by two researchers without knowledge of the group to which each post-test belonged. Each question was graded on a scale of 0-3, and regraded after discussion to resolve any differences of more than 1 point. The average of those two grades will be used in the results.

The topics covered in the experiment were relevant to students in two different phases of their learning cycle within the physics course. This experiment was performed on a weekend, 10 days before the first midterm exam. The material on superposition had already been lectured upon and also covered in homework, lab, and discussion section. The potential difference materials had been lectured upon, but homework, lab, and discussion sections on the topic had not yet occurred. Hence, students had not yet worked many problems in that area.

\section{RESULTS}

We now present results that show similarities and differences in how the students performed during and after the treatment period of the experiment. Figure 1 shows a box-and-whiskers plot of the time spent on each level, with the median reported in the black line in the center. The distribution of the time spent on each level was skewed due to several outliers in both groups that grew frustrated and were not able to proceed, and because of this the median was chosen as the best measure for central tendency. Both treatment groups spent similar amounts of time on each level. This is an interesting result given that the requirements and feedback for the two groups were quite different. One may have expected the mastery group to take more time, since they typically had to answer more questions, and had the higher standard of getting all questions correct on the first try. However, it appears that the after-level feedback that the mastery group received, including the solutions, compensated for the additional requirement. While the time spent was similar, looking into other facets of student behavior reveals how these two groups spent that time differently. 


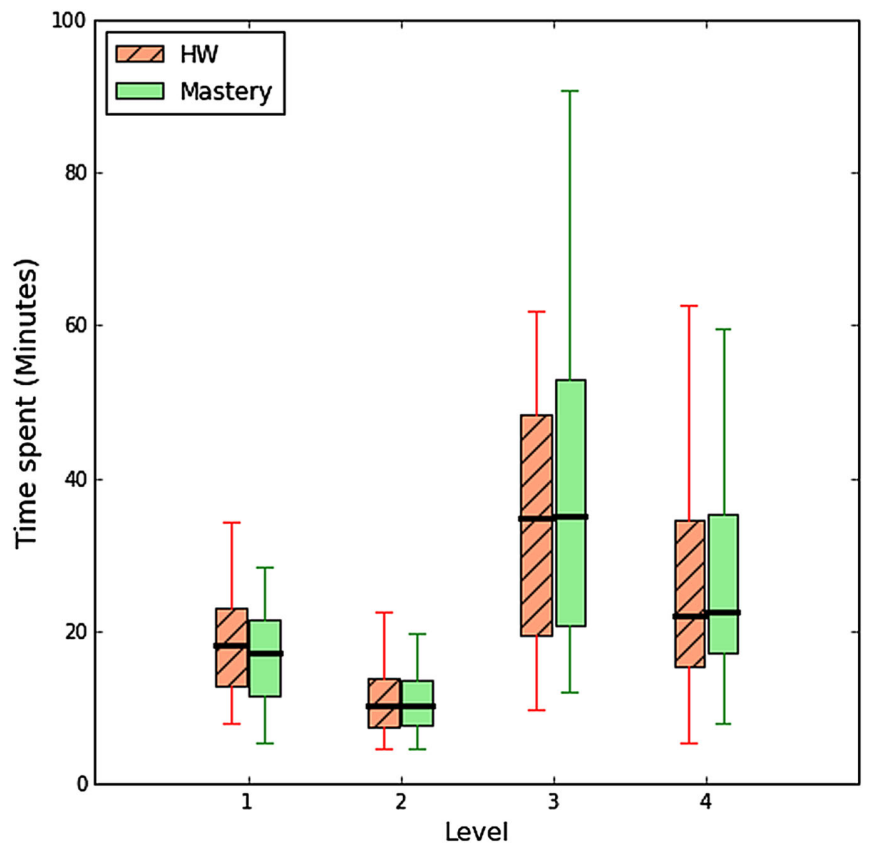

FIG. 1. Box-and-whiskers plot of time spent on each level in minutes. Median values are reported in the black line in the center of each box, and the boxes represent the interquartile range $(\mathrm{IQR}=\mathrm{Q} 3-\mathrm{Q} 1)$. Whiskers represent the most extreme value, for example, for the top whisker, within $1.5 \times \mathrm{IQR}+\mathrm{Q} 3$. Outliers are not shown. Groups spent similar amounts of time on each level.

Although students spent similar amounts of time on each level, the homework group submitted more answers than the mastery group. Figure 2 shows box plots for the number of submissions per question for each level for both groups. Box plots were again chosen due to the skewness of the data. For every level, the mastery group had a lower median number of submissions than the homework group, significantly lower for levels 2 and 4 ( $p<0.01$ via the Mann Whitney test). This is not surprising since the homework group was free to answer each question as many times as necessary. Since only the initial version of each question was used, students were not penalized for using trial and error. Students in the mastery group responded to the higher stakes of getting all four questions correct, and the possibility of one wrong answer resulting in four new questions to answer, by taking more time between submissions. One extreme example of trial and error behavior in the homework group was students choosing to increment their submissions. The homework system told students when they were off by a power of ten, and rather than calculate the correct order of magnitude of their answer, some students incremented that order of magnitude, one student going so far as to answer 93 times on one question. Trial and error was not a feasible strategy in the mastery group, since each wrong answer resulted in a new version of all questions in each level. That strategy, along with the heavily tailed nature of the submission data, precipitated

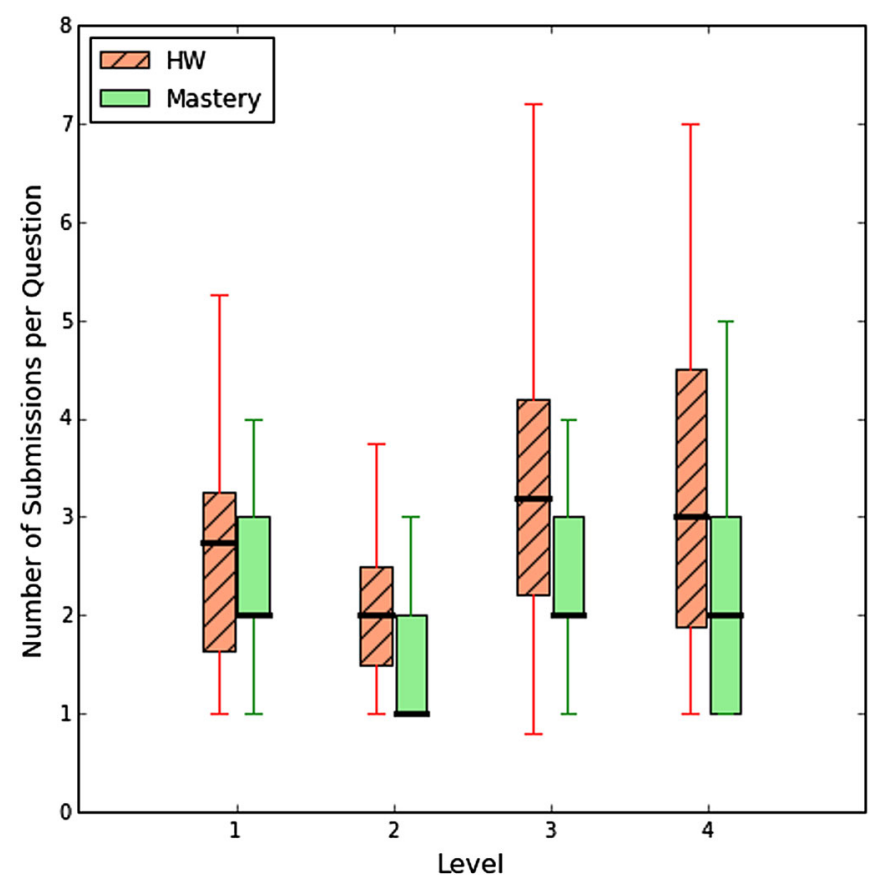

FIG. 2. Box plot of the number of submissions per question on each level. Median values are reported in the black line in the center of each box, and the boxes represent the interquartile range (IQR = Q3-Q1). Whiskers represent, for example, for the top whisker, most extreme value within $1.5 \times \mathrm{IQR}+\mathrm{Q} 3$. Outliers are not shown. The mastery group had fewer median submissions per question for all levels.

the choice to use medians instead of means in Fig. 2. Another interesting behavior arose in the mastery group, where some students skipped the first version of each level by quickly submitting an answer to all the questions then studying the solutions to those questions. The ability to effectively skip the first attempt at a level allows students who know they are novices at the skills being tested to go straight to the solution videos, using them as worked examples.

Another difference between the two groups is revealed in looking into performance on the first try for each question, keeping in mind the different conditions under which students attempted the questions. Figure 3 shows the fraction of students with a correct response after their 1st try for both groups on each question. The homework group tended to exhibit improvements between questions, whereas the mastery group improved between level attempts. For level 1, question 1, the mastery group outperformed the homework group. After answering the first question, the mastery group students did not get feedback, and moved on to attempt the rest of the questions for level 1, whereas the homework group got immediate correctness feedback, and could repeat the attemptfeedback cycle until they answered the question correctly. Students in the homework group could find a correct solution to question 1 before attempting question 2 . The 


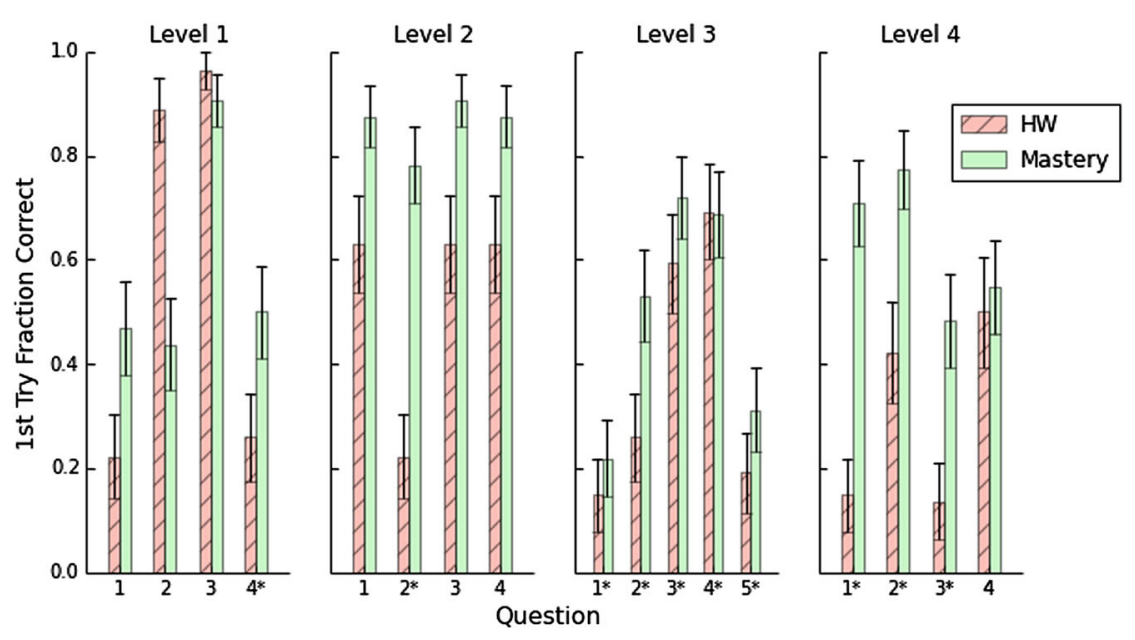

FIG. 3. Fraction of students with a correct response on their first try for each question. Asterisk indicates question had different formats for the two groups (multiple choice for the mastery and free response for the homework group).

strategies needed to correctly answer questions 1 and 2 for the first level were very similar. The homework group improved greatly from question 1 to 2 . The mastery group had no such feedback, and performed similarly on the two questions. Here it should be noted that the difference seen in question 4 for the first level is possibly due to the homework group having a free response format versus the mastery group having multiple choice (indicated by the asterisk in Fig. 3). ${ }^{1}$ The same format difference applies to level 2 question 2. For the other three questions in level 2, which had the same format, the mastery group significantly outperformed the homework group. This level used many skills covered in level 1 , so the increased performance of the Mastery group is probably due to the feedback given while students mastered level 1, suggesting the mastery style learned more from their work on level 1. For level 4, questions 1 and 2 were very similar, and the same pattern seen in level 1 was exhibited, where the mastery group performed the same on both questions, and the homework group improved from the first to the second question. While level 4 questions 1 and 2 had the format difference between the two groups, those questions are very similar to level 3 question 1. The homework group did not improve their score from level 3 question 1 to level 4 question 1, while the mastery group showed a very large improvement, suggesting the mastery group learned more from their

\footnotetext{
${ }^{1}$ Investigating the content of student answers in the homework group on free response questions and comparing them with the available answers in the multiple choice format does bring the score up on several of the questions shown in Fig. 3, but does not change any of the results noted here. For example, the score on level 2, question 2 for the homework group increases to $58 \%$ if we only accept the first answer submitted that matches a multiple choice answer for the homework group. Smaller increases are seen for level 3, question 2 and level 4, questions 1 and 2, but the conclusions noted are not changed.
}

experience in level 3. After the question and feedback portion of the experiment, students proceeded on to a post-test.

On the post-test the Mastery group $(16.0 \pm 1.2)$ significantly outperformed the HW group $(9.7 \pm 1.3$, $p<0.005)^{2}$ The results, in total and by question, are shown in Fig. 4. The Mastery group outperformed the homework (HW) group on all questions. The amount of difference between the study materials and the post-test varied with each question, with the most similar being question 8 , which was very similar to question 3 from level 4 of the instruction materials. On question 8 students were required to conduct an integral over 2 regions, one of which was inside a conductor. This was very close to activities required in the third and fourth levels. Question 8 also resulted in the most significant difference between the two treatment groups. Mastery group students also outperformed the homework group when required to combine what they learned about electric fields in planar geometries with what they learned about potential difference in spherical geometries, and transfer that to the topic of potential difference in planar geometries, evidenced by questions 2, 4, and 6. Question 5, which resulted in the smallest difference, asked students to work backwards from a given potential difference between two points and a given charge density on one sheet of charge to find the charge density on a second sheet. Students were trained on finding a charge density from a given electric field, but perhaps the combination of this skill with potential difference calculation was too difficult to grasp, and too different from the learning materials.

\footnotetext{
${ }^{2}$ Post-test results are significant whether evaluated using either grader's initial post-test grades or the averaged final grade.
} 


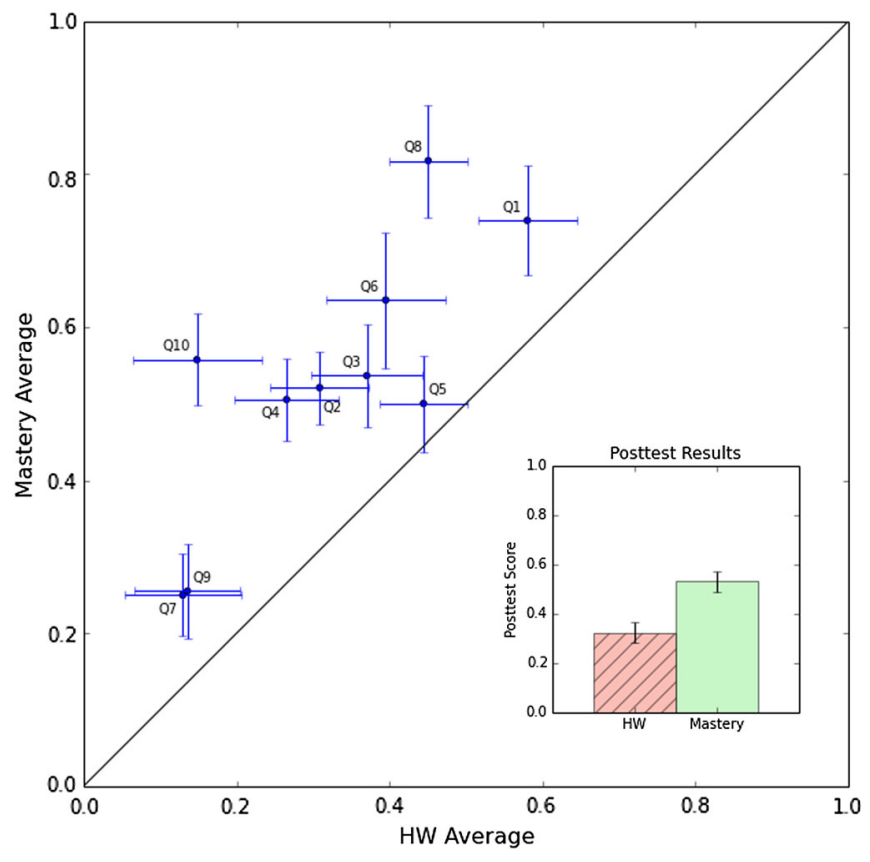

FIG. 4. Outset: Mastery group normalized average score HW group average for each question. Points above the line indicate the Mastery group outperformed the HW group. Inset: Normalized total post-test score average for both groups.

\section{DISCUSSION}

We performed an experiment to compare how students interact with and learn from two types of online homework, a mastery group that used delayed feedback coupled with narrated solutions, and a homework group that used materials simulating online homework systems. Students in the mastery group significantly outperformed the homework group on a post-test examining planar and spherical potential differences. On all questions, the Mastery group outperformed the Homework group, significantly on five of them, including questions that were similar to the testing materials, such as question 8 , and questions that required synthesizing planar electric fields and potential difference calculations, such as questions 2 and 4. Mastery learning combined with narrated animated solution videos resulted in more learning in a similar amount of time spent than when students were exposed to more traditional online immediate feedback systems. Successfully combining formative assessment, learning from multimedia worked examples, and mastery learning can result in more efficient learning for students.

In the homework group treatment we sought to simulate online immediate feedback homework systems by allowing students to try a question as many times as they would like. Students responded to the experiment conditions by optimizing their behavior to move forward quickly. Students in the homework group answered more frequently than the mastery group, which did not surprise us, given that there was no penalty for a wrong answer for the homework group, versus the penalty of having to start over again on a level for the mastery group. Some students took this to the extreme of quickly incrementing answers in hopes of stumbling upon the correct answer. The extremely low stakes of answering the immediate feedback style questions for the homework group may have encouraged students to trial and error behavior. There was no penalty for trying quick, easy strategies. Homework group students had access to notes as well as other experiment participants, but only made use of those resources after they reached an impasse in their work. For the mastery group, the higher stakes caused students to exhibit much different behavior. This was best exemplified in the visible stress we saw in students when they submitted their answers for grading, and the elation or frustration we saw when they saw their results. Students in the mastery group checked their answers before submitting, whereas the homework group did not. This added step of sense making may have helped students in the mastery group learn and understand the strategies they were employing.

The feedback conditions also measured learning in different sequences. Students in the homework group effectively treated each question like its own level, trying it again and again until a successful strategy was found, exhibited in the improvement between the first two questions on levels 1 and 4 . The mastery group's first try scores improved not between questions, but between levels, after they had either found or been shown and then found the correct strategy for a question. This delayed feedback with solutions may make students work more before seeing a positive result, but in the end resulted in a larger learning gain on the post-test.

This experiment uses the same questions and solutions as an earlier experiment conducted at the same institution [28]. That experiment used an $85 \%$ mastery threshold (meaning students could miss one question and still pass a level), and had 2 levels of 8 and 9 questions, one on planar electric fields and one on spherical potential differences, that were split into the 4 levels used in this experiment. We desired to send a stronger message to students in the experiment with the $100 \%$ pass threshold, and compensated for the stricter conditions by making the levels shorter, allowing students to focus on fewer skills at a given time, and answer fewer questions to pass a level. This had several consequences. While we did not collect data on student affect in this experiment, students frequently exhibited positive gestures when they mastered a level. More opportunities were presented for success with shorter, more numerous levels. We expected students to move through the materials in this experiment at a faster rate than in the prior experiment, but the total time spent remained similar. Students moved through the first two levels quicker in this version of the experiment than through the first level of the prior experiment. The $100 \%$ threshold seems to have slowed the students in this experiment down, particularly in the 3rd and 4th levels, 
which we suspect is due to the tricky and time consuming task of keeping track of the many negative signs in potential difference calculations, a fertile ground for careless errors. In the prior experiments, students had some leeway to make a mistake, since they could move forward while still getting one question wrong, but here they had to correctly answer an entire set of questions on the same try for each level. Frustration from repeated failure on the same question in a level is of concern, as students who are tired may become frustrated, hampering their ability to learn from the presented solutions, which in turn kept them from moving through the materials. Future creators of mastery materials should be wary of questions that lend themselves to algebraic errors, as possible points for major student frustration.

This experiment was conducted in one sitting in order to optimize recruitment of students. As a result, there was no delay between the instruction period of our experiment and the post-test. The successful application of the skills taught in the instructional period to new contexts in the post-test may have reflected the storage of those skills in short-term rather than long-term memory. Questions 1-7 on the posttest employed planar charges. The material that covered the fields in that geometry were taught in levels 1 and 2, and the skills learned had to persist through the final two levels. For the mastery group, their performance on questions 1-7 was closer to that of the homework group than for questions 8-10, which may indicate that student knowledge of planar electric fields decayed quickly in the intervening instructional time of the final two levels. Those final two levels were followed immediately by the post-test, so the high score for the mastery group on question 8 , the question most similar to the instructional materials, may be a reflection of the immediacy of the post-test. This could have been tested by inverting the order of the mastery levels, where one might expect that the mastery students would further outperform the homework group on the planar geometry questions because the post-test would not have the intervening instruction in a spherical geometry. The persistence of the knowledge learned could also be better tested by giving the post-test a week after the instructional period, which would give the short-term memory of the students ample time to forget the skills taught, forcing students to recall the information from long-term memory. Still, the mastery group did outperform the homework group significantly on the planar geometry questions, which required that knowledge of planar electric fields to persist through the spherical geometry instruction, as well as combining that knowledge of the fields with the potential difference calculation skills taught in those final two levels.

Several aspects of this learning treatment remain to be investigated. This experiment was conducted using volunteers that chose to spend their time on a weekend studying physics. These students do not represent the class as a whole, as $~ 90 \%$ of the course did not sign up to participate. While both groups of students came from this volunteer pool, these students were probably more dedicated and focused to the learning task than the average student, and that may have increased the advantage of the mastery group over the homework group. Students in the mastery group in our experiment watched a high fraction of the solutions to questions they missed. This may not be the case if students are not volunteering to take part in a clinical experiment. Differences between versions of a level were kept as small as possible in this experiment. Larger differences between level versions may result in frustrated students, but could also challenge students and result in larger learning. While we did not see any of the incrementing behavior seen in the homework group, students may find other ways to sidestep the requirements of a mastery assignment. We did not collect data on student affect in this experiment, but given the possibilities for student frustration, this should be investigated in the future. We are implementing mastery learning on a course-wide scale for an introductory course to see how students interact with these materials in a nonclinical environment. We will present results in a future publication.

\section{ACKNOWLEDGMENT}

This work was partially funded by National Science Foundation DUE Grant No. 0817185.
[1] https://webassign.com/corporate/about-us/ Accessed 11/ $19 / 2014$.

[2] J. Mestre, D. M. Hart, K. A. Rath, and R. Dufresne, The effect of web-based homework on test performance in large enrollment introductory physics courses, J. Comput. Math. Sci. Teach. 21, 229 (2002).

[3] S. Bonham, R. Beichner, and D. Deardorff, Online homework: Does it make a difference?, Phys. Teach. 39, 293 (2001).
[4] A. Pascarella, in Proceedings of the NARST 2004 Annual Meeting, Vancouver, BC, 2004 (unpublished) (http://www .loncapa.org/papers/204416ProceedingsPaper.pdf).

[5] J. R. Anderson, A. T. Corbett, K. R. Koedinger, and R. Pelletier, Cognitive tutors: Lessons learned, J. Learn. Sci. 4, 167 (1995).

[6] A. C. Butler, J. D. Karpicke, and H. L. Roediger III, The effect of type and timing of feedback on learning from multiple-choice tests, J. Exp. Psychol. Appl. 13, 273 (2007). 
[7] V. J. Shute, Focus on Formative Feedback, Rev. Educ. Res. 78, 153 (2008).

[8] A. C. Butler and H. L. Roediger III, Feedback enhances the positive effects and reduces the negative effects of multiple-choice testing, Mem. Cogn. 36, 604 (2008).

[9] X. Zhu and H. A. Simon, Learning mathematics from examples and by doing, Cognit. Instr. 4, 137 (1987).

[10] M. W. Carroll, Using worked examples as an instructional support in the algebra classroom, J. Educ. Psychol. 86, 360 (1994).

[11] M. T. H. Chi, M. Bassok, M. W. Lewis, P. Reimann, and R. Glaser, Self-explanations: How students study and use examples in learning to solve problems, Cogn. Sci. 13, 145 (1989).

[12] P. L. Pirolli and J. R. Anderson, The role of learning from examples in the acquisition of recursive programming skills, Canadian J. Psychol. 39, 240 (1985).

[13] J. R. Anderson, R. Farrell, and R. Sauers, Learning to program in LISP, Cogn. Sci. 8, 87 (1984).

[14] J. Sweller, Cognitive load during problem solving: Effects on learning, Cogn. Sci. 12, 257 (1988).

[15] J. Sweller and P. Chandler, Evidence for cognitive load theory, Cognit. Instr. 8, 351 (1991).

[16] S. Kalyuga, P. Ayres, P. Chandler, and J. Sweller, The expertise reversal effect, Educ. Psychol. 38, 23 (2003).

[17] W. G. Chase and H. A. Simon, Perception in chess, Cogn. Psychol. 4, 55 (1973).

[18] M. Ward and J. Sweller, Structuring effective worked examples, Cognit. Instrum. 7, 1 (1990).

[19] R. E. Mayer, Multimedia Learning (Cambridge University Press, Cambridge, England, 2001).

[20] W. Schnotz, Towards an integrated view of learning from text and visual displays, Educ. Psychol. 14, 101 (2002).

[21] T. Stelzer, G. Gladding, J. Mestre, and D. T. Brooks, Comparing the efficacy of multimedia modules with traditional textbooks for learning introductory physics content, Am. J. Phys. 77, 184 (2009).

[22] Z. Chen, T. Stelzer, and G. Gladding, Using multimedia modules to better prepare students for introductory physics lecture, Phys. Rev. ST Phys. Educ. Res. 6, 010108 (2010).

[23] B. S. Bloom, Learning for Mastery, Evaluation Comment. 1, 1 (1968) (UCLA-CSEIP).

[24] T. R. Guskey and S. L. Gates, A synthesis of research on group-based mastery learning programs, in Annual Meeting of the American Educational Research Association, Chicago, 1985, http://eric.ed.gov/?id=ED262088.

[25] C. L. C. Kulik, J. A. Kulik, and R. L. Bangert-Drowns, Effectiveness of mastery learning programs: A metaanalysis. Rev. Educ. Res. 60, 265 (1990).

[26] J. D. Karpicke and H. L. Roediger, The critical importance of retrieval for learning, Science 319, 966 (2008).

[27] H. L. Roediger, D. A. Gallo, and L. Geraci, Processing approaches to cognition: The impetus from the levels-ofprocessing framework, Memory 10, 319 (2002).

[28] P. Black and D. Wiliam, Assessment and classroom learning, Assess. Educ. Princ. Pol. Pract. 5, 7 (1998).

[29] N. Schroeder, G. Gladding, B. Gutmann, and T. Stelzer, Narrated animated solution videos in a mastery setting, Phys. Rev. ST Phys. Educ. Res. 11, 010103 (2015).

[30] Available at http://www.techsmith.com/jing.html.

[31] Z. Chen and G. Gladding, How to make a good animation: A grounded cognition model of how visual representation design affects the construction of abstract physics knowledge, Phys. Rev. ST Phys. Educ. Res. 10, 010111 (2014).

[32] See Supplemental Material at http://link.aps.org/ supplemental/10.1103/PhysRevSTPER.11.010114 for representative screenshots of the final frames of two solution videos, the 15 question sets used in this experiment, and the post-test used in this experiment. 